\title{
Value of the Perineal Ultrasound in the Diagnosis of Anterior Urethral Stricture in Male Patient
}

\author{
Firas Abdullah Noori ${ }^{1}$, Ali Abdu Baqi Ali ${ }^{2}$, Riyadh Adel Abdulazeez ${ }^{3}$ \\ ${ }^{1}$ Assist. Prof., FICMS, Department of Radiology, College of Medicine, University of Thj-Qar, Iraq, ${ }^{2}$ Assist. \\ Prof., FICMS, Department of Urology, College of Medicine, University of Thj-Qar, Iraq, ${ }^{3}$ Assist. Prof., FICMS, \\ Department of Radiology, College of Medicine, University of Thj-Qar, Iraq
}

\begin{abstract}
Objectives: Determine the role of perineal ultrasound in the diagnosis of anterior urethral strictures and assessment of peristenotic fibrosis.

Material and Method: Thirty healthy adult male and thirty patients with suspected urethral stricture have had urethral ultrasound after distension of urethra with a normal saline. The thirty patents also underwent retrograde urethrogram (RUG).

Results: Average diameters healthy urethra were $11.6+/-3.1 \mathrm{~mm}$ at penile level, $11.2+/-2.7 \mathrm{~mm}$ at the bulbar level, and $9.7+/-2.88 \mathrm{~mm}$ at the bulbomembranous level. The periurethral tissue thickness averaged $2.7+/-0.5 \mathrm{~mm}$ at penile level, from $3.5+/-0.65 \mathrm{~mm}$ to bulbar level and $3.8 \pm 0.6 \mathrm{~mm}$ at the bulbomembranous level. Ultrasound made the diagnosis of 31 stenosis or $96.87 \%$. On ultrasound, the length of the stenosis was significantly longer than that seen on retrograde urethrogram with a significant difference between the two techniques $(\mathrm{P}=0.045)$. There is also a significant difference in diameter of urethral strictures between the two techniques. The thickness of the periurethral tissue at the level urethral stenosis was more than that measured in a healthy zone in all cases, whatever the urethral portion concerned. There was not no correlation between periurethral tissue thickness and degree of stenosis.
\end{abstract}

Conclusion: Urethral ultrasound is a method that allows us to make the diagnosis of urethral stricture and to assess periurethral fibrosis. It could validly replace retrograde urethrogram in diagnosing anterior urethral stricture.

Keywords: Perineal ultrasonography. Urethral stricture. Retrograde urethrogram.

\section{Introduction}

Urethral stenosis is the decrease of the caliber of the urethra, causing a resistance to the antitrade flow of urine $^{1}$. Stricture of male anterior urethra is a common pathology and may be congenital or acquired in origin ${ }^{2}$. It has a therapeutic problem which is the risk of recurrence related to the development of a peristenotic fibrosis in the spongy tissue ${ }^{3}$. A precise therapeutic preassessment taking into account the characters of the urethral stenosis and peristenotic tissue appears so indispensable ${ }^{3}$. The usual investigation of diagnosing urethral stricture were based on retrograde urethrogram which have limits in evaluation of the exact length of the urethral stricture and they cannot appreciate the associated spongy fibrosis ${ }^{3,4}$.
Ultrasound could be a good alternative to them due to the possibility of evaluation both the urethral stenosis and the peristenotic tissue (3-4). Our purpose is determining the value of ultrasound in the diagnosis of urethral stricture and in the appreciation of peristenotic fibrosis.

\section{Patients and Method}

We conducted a prospective study over one-year period that has consisted of comparing the ultrasound of the anterior urethra to the retrograde urethrogram (RUG) in adults. Sixty adult men were recruited from the urology department and have been divided into two groups. Thirty men were considered healthy without trouble in micturition or antecedent of urine pathology 
have undergone Urethral ultrasound. The ultrasound study of the normal urethra made us familiar with normal ultrasound anatomy before studying the stenotic urethra. Thirty patients suspected of having a urethral stenosis have undergone RUG and urethral ultrasound performed on same day.

Technical: At retrograde urethrogram: number, degree of stenosis, length and gauge stenosis were appreciated each time on the view that unfolded it best. The length and gauge were evaluated by direct measurements on radiographic images. The degree of stenosis was obtained by dividing the diameter of the residual lumen in narrowed area on the diameter of the healthy zone downstream. Urethral Ultrasound were performed using an ultrasound General Electrics Logiq E9 machine with an ML 6-15 probe. A specialized radiologist who did not know the results of RUG.

\section{Normal}

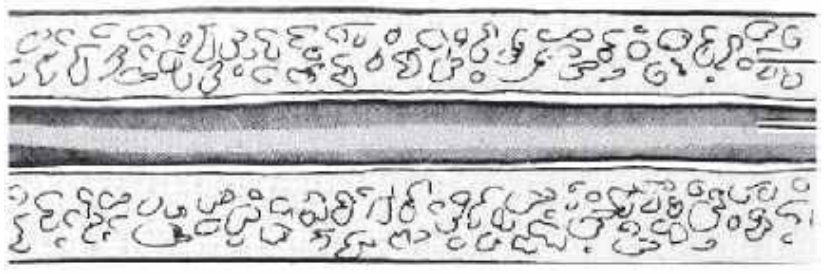

\section{Mild $<1 / 3$ Lumen occluded}

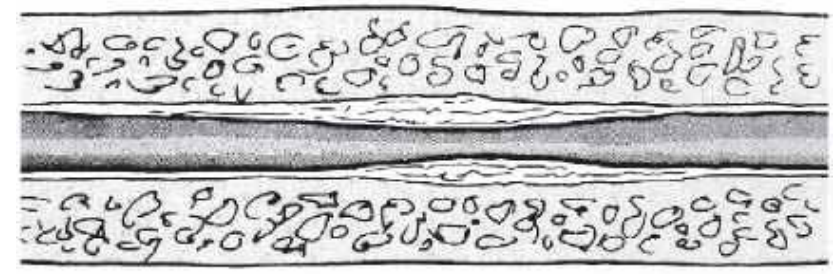

\section{Moderate 1/3-1/2 Lumen occluded}

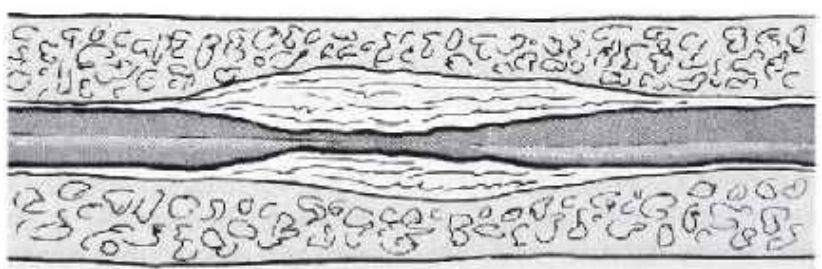

\section{Severe $>1 / 2$ Lumen occluded}

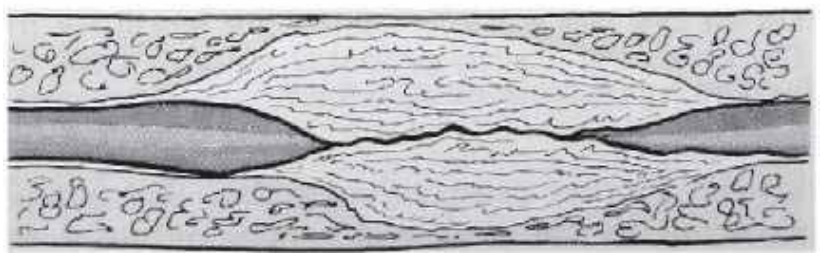

Fig (1): McAninch Ultrasound classification of the degree of urethral stricture
The patient was lying supine; the urethra was distended by a retrograde perfusion of normal saline. The penile urethra has been visualized by a ventral approach, the bulbar urethra was visualized by transscrotal, and bulbo-membranous urethra was visualized transperineally. The urethral strictures were evaluated by direct measurements on the screen in their following aspects: topography, number, degree of stenosis, length, caliber. The echostructure and the corpus spongiosus peristenotic thickness were compared to those of the adjacent normal area, to appreciate peristenotic fibrosis. We adopted the classification by MacAninch ${ }^{4}$ of the degrees of stenosis. According to this, a mild stenosis is less than $33 \%$, moderate between 33 and $50 \%$ and severe stenosis is greater than $50 \%$. Examination ended with suprapubic route ultrasound of the bladder, prostate and seminal vesicles. Analysis was performed using IBM SPSS v 23.0 software. The paired t- test and correlation coefficients were calculated. In all tests, $p$ values of $\leq 0.05$ were considered significant.

\section{Results}

The average age of the 30 healthy subjects was $39.77+/-9.5$ years old. Average diameters healthy urethra were $11.6+/-3.1 \mathrm{~mm}$ at penile level, 11.2 $+/-2.7 \mathrm{~mm}$ at the bulbar level and $9.7+/-2.88 \mathrm{~mm}$ at the bulbomembranous level. The periurethral tissue thickness averaged $2.7+/-0.5 \mathrm{~mm}$ at penile level, from $3.5+/-0.65 \mathrm{~mm}$ to bulbar level and $3.8 \pm 0.6 \mathrm{~mm}$ at the bulbomembranous level. The average age of 30 patients suspected to have urethral stricture was $44.8+/-7.71$ years old.

On RUG, we observed 32 urethral strictures including 28 single and two double strictures. At the ultrasound radiologist reported 31 urethral strictures, $96.87 \%$ of which 29 single and one double strictures. Because in one patient who had two strictures (bulbar and penile), ultrasound had detected only the penile one.

At RUG, the types of urethral strictures were the following: 5 penile (15.63\%), 21 bulbar (65.62\%), 6 bulbo-membranous (18.75\%). Locations on ultrasound were identical but one bulbar stricture less (figure 2). Urethral stricture causes were traumatic in 6 cases, 20\% and post-infectious in 24 cases, $80 \%$.

The length of the strictures was compared in an accurate way between the two techniques RUG and ultrasound. Of 26 strictures, the length of urethral stenosis measured on ultrasound is greater than that seen 
on RUG. Of 6 strictures, the length is the same between the two techniques. At RUG, strictures ranged from 2 to $14 \mathrm{~mm}$ with an average of $5.96+/-3.05 \mathrm{~mm}$. On ultrasound the length of the strictures varied from 3 to $25 \mathrm{~mm}$ with an average of $9.25+/-5.9 \mathrm{~mm}$, there was a significant difference between the two techniques $(\mathrm{P}=$ 0.045). As for the caliber, it varied from 1 to $7 \mathrm{~mm}$ at the RUG, while from 1 to $12 \mathrm{~mm}$ at ultrasound with averages of $3.1+/-2.2 \mathrm{~mm}$ and $4.6+/-1.9 \mathrm{~mm}$ respectively with a significant difference $(P=0.05)$. The comparison of the degree of stenosis with RUG and ultrasound did not show significant difference $(\mathrm{P}=0.66)$. The minimum values were $12 \%$ and $15 \%$ at RUG and ultrasound and the maximum $70 \%$ and $80 \%$ respectively (table 1 ). The thickness of the periurethral tissue (TPU) at the level of urethral strictures was greater to that measured in a healthy zone in all cases, whatever the portion urethral concerned. This thickness averaged $6.9+/-2.05 \mathrm{~mm}$ at the level of penis, $6.3+/-1.67 \mathrm{~mm}$ at the level bulbar urethra and $6+/-1.8 \mathrm{~mm}$ at the bulbomembranous level. The peristenotic fibrosis had more echogenicity than healthy periurethral tissue but there was no correlation between periurethral tissue thickness and degree stenosis.

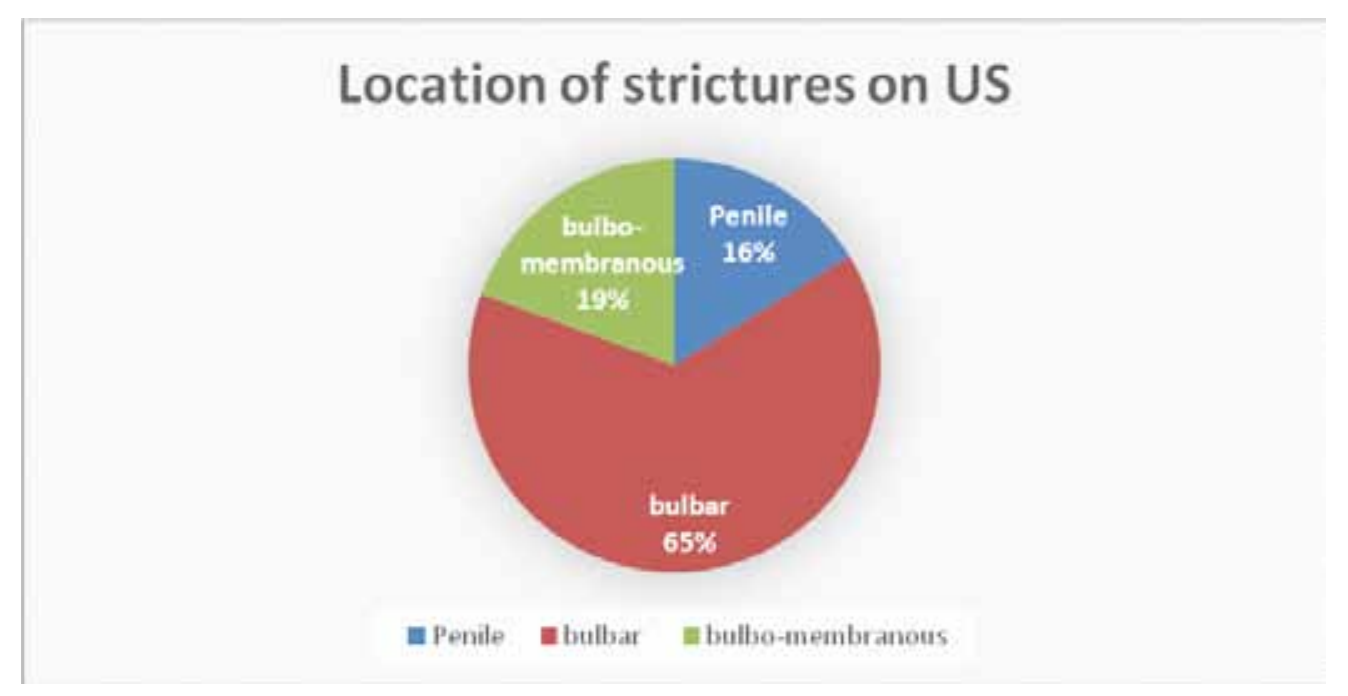

Figure (2): shows the percentage of strictures according to their location that seen on US

Table (1): shows the comparison between RUG and Ultrasonography

\begin{tabular}{|l|c|c|c|}
\hline Parameter & RUG & Ultrasonography & P-value \\
\hline Average of length of stricture & $5.96+/-3.05 \mathrm{~mm}$ & $9.25+/-5.9 \mathrm{~mm}$ & 0.045 \\
\hline Average of caliber of stricture & $3.1+/-2.2 \mathrm{~mm}$ & $4.6+/-1.9 \mathrm{~mm}$ & 0.05 \\
\hline Average of degree of stenosis & $12-70 \%$ & $15-80 \%$ & 0.66 \\
\hline
\end{tabular}

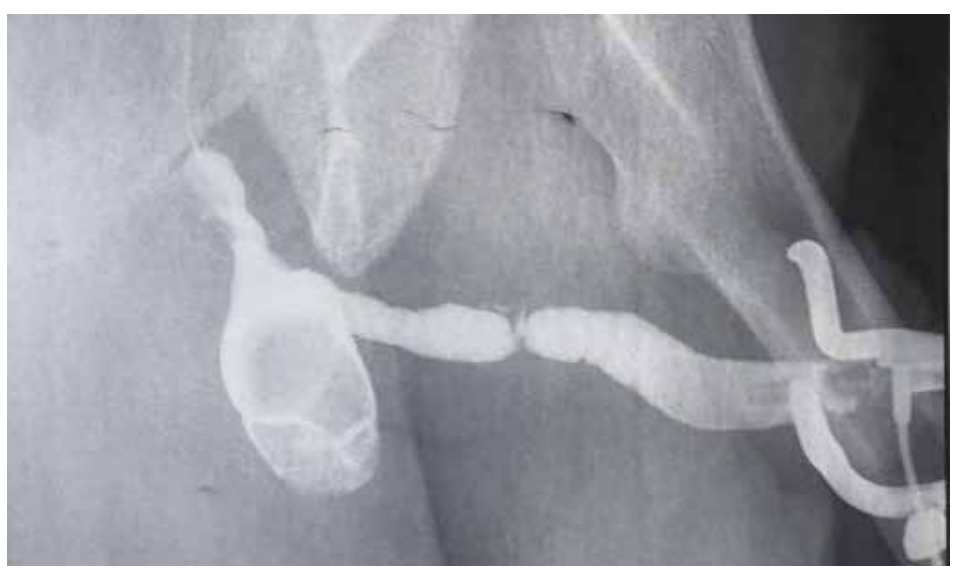

Figure (2): RUG showing urethral stricture and diverticulum 


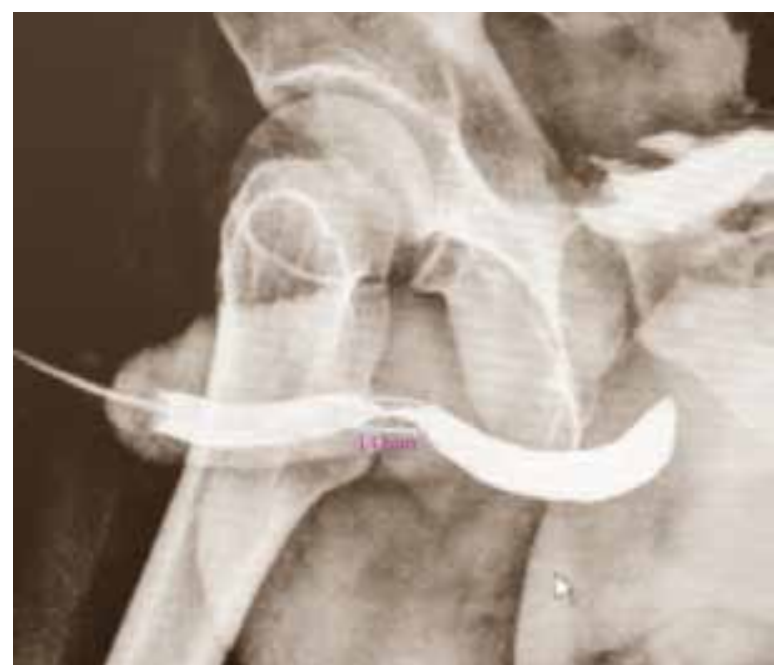

Figure (3): RUG showing urethral stricture $(14 \mathrm{~mm})$ in length

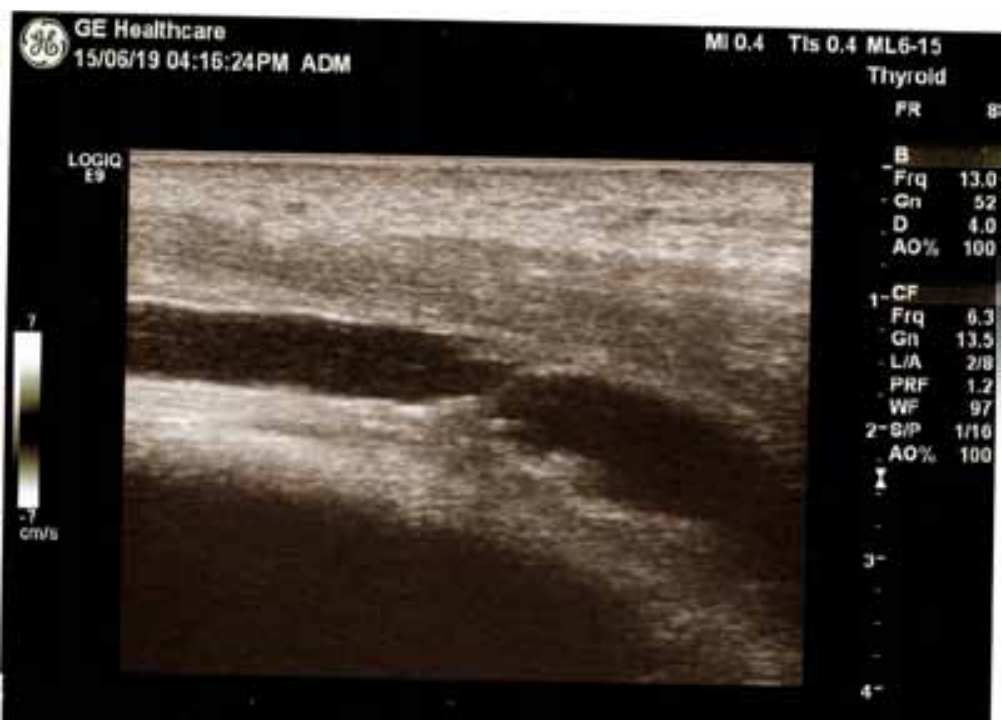

Figure (4): perineal US showing urethral stricture

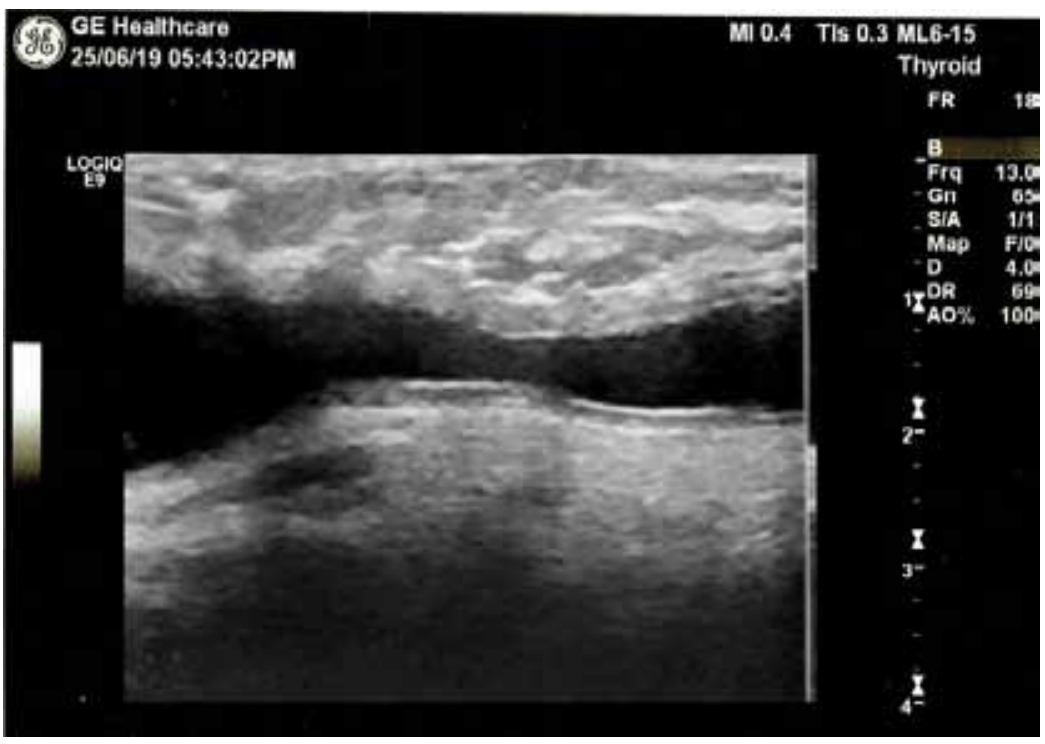

Figure (5): perineal US showing urethral stricture 


\section{Discussion}

The average age of patients with urethral stricture was $44.8+/-7.71$ years has been in line with the data from the Quirassy study ${ }^{6}$. Preponderance of the infectious etiology was observed by many authors. Ultrasound diagnosed with urethral stenosis in $96.87 \%$, these results are comparable those of Gluck's study ${ }^{8}$ in which two bulbar urethral strictures were undetected without having a precise reason. In our study undiagnosed stenosis could be explained in that the patient presented a double stenosis and only the stenosis the tighter was individualized. This would be overrided with more experience of examining urethral strictures. We found that the lengths of urethral strictures were significantly greater in ultrasound than RUG, our results are consistent with those in Das [9] while differs from the results of Pierredon- Fulongna ${ }^{3}$, in which the lengths on ultrasound and UCAM were substantially equal. Our ultrasound measurements were not compared to the surgical results. But studies which have compared the length of the urethral stenosis determined by ultrasound and by RUG to the urethroplasty results showed better ultrasound reliability $4,5,10$. Ultrasound measurements seemed more reliable because they have been carried out directly on the exact stenotic area without radiological magnification or distortion of the image. In our study there was a concordance of the topography of urethral strictures between RUG and ultrasound except for one bulbar stenosis. The predominance of the bulbar seat (65.62\%) in our work was observed by other authors ${ }^{1,4,7,10}$. This predominance could be due to stagnation of urethral gland secretions at this level according to Thoumas ${ }^{10}$. We did not have observed a significant difference between degree of stenosis measured on RUG and ultrasound as in Pierredon-Foulongne study ${ }^{3}$. On ultrasound, a periurethral tissue thickening in the portions where there were stenosis has been demonstrated in all our patients. However, we could not distinguish peristenotic tissue from the fibrosis properly. The prognostic value of the echogenicity of the peristenotic tissue could not be specified in the absence of comparison with the followup imaging of the patients. Several authors $(3-5,10)$ noted in their studies that the visualization of the fibrosis was inconstant with its ultrasound appearance, because it was hyper or isoechoic for some ${ }^{3,10}$ and hypoechoic for others ${ }^{5}$. The use of a higher frequency probe would provide a better definition of peristenotic structures according to the study of Garcia ${ }^{11}$. Magnetic resonance imaging can highlight any evidence of an inflammation of the urethra in the form of a diffuse thickening of the
TPU which appears as an intermediate signal intensity in T2 weighed imaging ${ }^{12,13}$.

\section{CONCLUSSION}

Urethral ultrasound is an easy accessible method of diagnosing urethral stricture with a greater safety than retrograde urethrography and voiding cystourethrogram. Ultrasound can reliably replace them especially in postinfectious etiologies after proper training of the operator. Ultrasonography has more value than RUG in assessment of length and caliber of the anterior urethral stricture. Also has advantage in assessing the periurethral tissue thickness (TPU).

Financial Disclosure: There is no financial disclosure.

Conflict of Interest: None to declare.

Ethical Clearance: All experimental protocols were approved under the Department of Radiology and all experiments were carried out in accordance with approved guidelines.

\section{References}

1. Tritschler S, Roosen A, Füllhase C, Stief CG, Rübben H. Urethral Stricture. Deutsches Aerzteblatt. 2013 Mar; 110(13): 220-226.

2. Gupta, A., Gupta, A., \& Sharma, K. (2015). Comparative Evaluation Of Sonourethrography (Sug) And Retrograde Urethrography (Rgu) In Detection Of Urethral Strictures. Journal of Evolution of Medical and Dental Sciences, 2015 04(18), 3026-3031.

3. Helen L. Nicholson, Yasser Al-Hakeem, Javier J. Maldonado,Vincent Tse. Management of bladder neck stenosis and urethral stricture and stenosis following treatment for prostate cancer. Transl Androl Urol. 2017 Jul; 6(Suppl 2): S92-S102.

4. Maciejewski C, Rourke K. Imaging of urethral stricture disease. Translational andrology and urology; 2015, Feb; 4(1): 2-9.

5. Gyupta S, Majumdar B, Tiwari A, Gupta KR, Kumar A, Gujral RB. Sonouretrography in the evaluation of anterior uretral strictures: correlation with radiographic uretrography. JCU 1993;21: 231-9.

6. Heidenrich A, Derschum W, Bonfig R, Wilbert DM. Ultrasound in the evaluation of uretral stricture 
disease: a prospective study in 175 patients. $\mathrm{Br} \mathrm{J}$ Urol 1994; 74:93-8.

7. Quirassy S. Étude rétrospective du rétrécissement urétral au CHU Ignace-Deen: janvier 1985-octobre 1990. Journal d'urologie 1992;2:101-4.

8. Gluck CD, Bundy AL, Fine C, Loughlin KR, Richie JP. Sonographic urethrogram: comparaison to roentgenographic techniques in 22 patients. $\mathrm{J}$ Urol 1988;140: 1404-8.

9. Das S. Ultrasonographic evaluation of uretral stricture disease. Urology 1992;3: 237-42.

10. Thoumas D, Sibert L, Marlière F et al. Échographie de l'urètre: valeur pronostique de l'échostructure de la fibrose péristénotique. Étude prospective sur 33 patients. Progrès en urologie 1997;7 :628- 32.

11. Garcia-Medina V, Berna JD, Lherena J, GarciaMedina J, Genove JL. Uretral sonography in the diagnosis of penile and bulbar uretral stenosis. Eur J Radiol 1992;14:31-6.

12. Jeong-ah Ryu and Bohyun Kim. MR Imaging of the Male and Female. Urethra Radiographics. 2001;21:1169-85.

13. Pretorius ES, Evan SS, Parvati R and Marc PB. Helping the Urologist. MR Imaging of the Penis. Radiographics 2001;21:283-98. 